\title{
Outer edges of debris discs
}

\section{How sharp is sharp?}

\author{
P. Thébault ${ }^{1,2}$ and $\mathrm{Y} . \mathrm{Wu}^{3}$ \\ 1 Stockholm Observatory, Albanova Universitetcentrum, 10691 Stockholm, Sweden \\ 2 Observatoire de Paris, Section de Meudon, 92195 Meudon Principal Cedex, France \\ e-mail: philippe.thebault@obspm.fr \\ 3 Department of Astronomy and Astrophysics, University of Toronto, 50 St.George Street, Toronto, ON M5S 3H4, Canada
}

Received 23 November 2007 / Accepted 29 January 2008

\section{ABSTRACT}

\begin{abstract}
Context. Rings or annulus-like features have been observed in most imaged debris discs. Outside the main ring, while some systems (e.g., $\beta$ Pictoris and AU Mic) exhibit smooth surface brightness profiles (SB) that fall off roughly as $\sim r^{-3.5}$, others (e.g. HR 4796A and HD 139664) display large drops in luminosity at the ring's outer edge and steeper radial luminosity profiles.

Aims. We seek to understand this diversity of outer edge profiles under the "natural" collisional evolution of the system, without invoking external agents such as planets or gas.

Methods. We use a multi-annulus statistical code to follow the evolution of a collisional population, ranging in size from dust grains to planetesimals and initially confined within a belt (the "birth ring"). The crucial effect of radiation pressure on the dynamics and spatial distribution of the smallest grains is taken into account. We explore the dependence of the resulting disc surface brightness profile on various parameters.

Results. The disc typically evolves toward a "standard" steady state, where the radial surface brightness profile smoothly decreases with radius as $r^{-3.5}$ outside the birth ring. This confirms and extends the semi-analytical study of Strubbe \& Chiang (2006, ApJ, 648, 652) and provides a firm basis for interpreting observed discs. Deviations from this typical profile, in the form of a sharp outer edge and a steeper fall-off, occur for two "extreme" cases: 1) when the birth ring is so massive that it becomes radially optically thick for the smallest grains. However, the required disc mass is probably too high here to be realistic; 2) when the dynamical excitation of the dust-producing planetesimals is so low $(\langle e\rangle$ and $\langle i\rangle \leq 0.01)$ that the smallest grains, which otherwise dominate the optical depth of the system, are preferentially depleted. This low-excitation case, although possibly not generic, cannot be ruled out by observations for most systems, .

Conclusions. Our "standard" profile provides a satisfactory explanation for a large group of debris discs that show smooth outer edges and $S B \propto r^{-3.5}$. Systems with sharper outer edges, barring other confining agents, could still be explained by "natural" collisional evolution if their dynamical excitation is very low. We show that such a dynamically-cold case provides a satisfactory fit to the specific HR4796A ring.
\end{abstract}

Key words. stars: circumstellar matter - stars: individual: $\beta$ Pictoris - stars: individual: HR4796A - planetary systems: formation

\section{Introduction}

\section{1. the ubiquity of ring-like features}

Dusty debris discs have been detected by their infrared excess around $\sim 15 \%$ of nearby main sequence stars (e.g. Backman \& Paresce 1993). More than a dozen of these discs have also been imaged, mainly in scattered light, since the initial observation of the $\beta$ Pictoris system by Smith \& Terrile (1984). One unexpected result from these images is that almost no system displays a smooth extended radial profile: the usual morphology is the presence of rings (or annuli) where the bulk of the dust population is located. This ring morphology is in fact so common that Strubbe \& Chiang (2006) pointed out that the debris disc phenomenon could more appropriately be renamed debris "ring" phenomenon. Even for the archetypal debris "disc" $\beta$ Pictoris, which has been imaged from 5 to a few thousand AU, the bulk of the dust is probably concentrated in a rather narrow region between 80 and 120 AU (e.g. Augereau et al. 2001). One of the few systems actually resembling an extended "smooth" disc might be Vega, for which Spitzer mid-infrared images show a rather smooth radial luminosity profile ( $\mathrm{Su}$ et al. 2005). At the other end of disc morphologies, among the most striking ring features are the ones around HR4796 (e.g. Jayawardhana et al. 1998; Koerner et al. 1998; Schneider et al. 1999), HD 139664 (Kalas et al. 2006), and Fomalhaut (Kalas et al. 2005).

The characteristics that most differentiates one ring-like system from the other is the sharpness of the luminosity drop at the inner and outer edges of the rings. We shall in this paper focus on the outer edge issue, for which systems can be basically divided into two categories (see also Kalas et al. 2006):

- "Sharp edge" rings, displaying abrupt surface brightness drops, sometimes as steep as $r^{-8}$ beyond the ring. The most representative members of this group are HR4796A and Fomalhaut.

- "Smooth edge" rings, with no sharp outer edge and a surface brightness drop beyond the ring in $r^{-3}$ or $r^{-4}$. The most famous examples are here $\beta$ Pic and AU Mic

See Table 1 for a list of outer-edge profiles for several resolved debris discs. 
Table 1. Geometry and surface brightness profile for a selection of debris disc systems resolved in scattered light images.

\begin{tabular}{lcccc}
\hline \hline System $^{a}$ & Orientation & Detected radial extent $^{b}$ & ${\text { Surface brightness } S B \propto r^{\alpha}}^{\alpha}$ & Reference \\
\hline HD 53143 & $\sim$ face-on & $55-110 \mathrm{AU}$ & $\alpha \sim-3$ & Kalas et al. (2006) \\
$\beta$ Pic & edge-on & $127-193 \mathrm{AU}$ & $\alpha \simeq-3$ & Golimovski et al. (2006) \\
& & $193-258 \mathrm{AU}$ & $\alpha \simeq-4$ & \\
HD 32297 & edge-on & $80-170 \mathrm{AU}$ & $\alpha \sim-3.5$ & Schneider et al. (1999) \\
& & $170-350 \mathrm{AU}$ & $\alpha \sim-3$ (averaged over the 2 ansae) & \\
AU Mic & edge-on & $32-210 \mathrm{AU}$ & $\alpha \sim-3.8$ (averaged over the 2 ansae) & Fitzgerald et al. (2007) \\
HD139664 & edge-on & $83-109 \mathrm{AU}$ & $\alpha \sim-4.5$ & Kalas et al. (2006) \\
HD 107146 & $\sim$ face-on & $130-185 \mathrm{AU}$ & $\alpha \sim-4.8 \pm 0.3$ & Ardila et al. (2004) \\
HD 181327 & face on & $100-200 \mathrm{AU}$ & $\alpha \sim-5$ & Schneider et al. (2006) \\
Fomalhaut & $6^{\circ}$ inclination & $140-160 \mathrm{AU}$ & $\alpha \sim-6.1$ & Kalas et al. (2006) \\
HR 4796A & $7^{\circ}$ inclination & $70-120 \mathrm{AU}$ & $\alpha \sim-7.5$ & Wahhaj et al. (2005) \\
\hline
\end{tabular}

${ }^{a}$ For many of these systems, many additional features, i.e., warps, clumps, etc., have been observed (it is especially true for $\beta$ Pictoris) but we focus here on the main issue of average radial profiles.

${ }^{b}$ The radial extents and surface brightness profiles are given for regions beyond the likely "birth ring" - the region where scattering luminosity peaks and where most parent bodies are believed to reside (see Sect. 2).

The presence of ring features is commonly attributed to some sculpting mechanisms including, in particular, the presence of a massive planet (see, for instance, Quillen 2007a; for Fomalhaut, Freistetter et al. 2007; for $\beta$ Pictoris, Wyatt et al. 1999; for HR4796A, or the more general study of Moro-Martin $\&$ Malhotra 2005). The gravitational effect of such a planet can truncate or create gaps in the disc, either directly on the dust particles or indirectly on the planetesimals that produce these particles.

\section{2. outer edges}

Nevertheless, while planets may be a natural and easy explanation for sculpting the inner edges, outer edges are a different problem. They are difficult to explain in the light of one well established fact about debris discs, i.e. that the observed dust is not primordial but steadily produced by collisions, through a collisional cascade starting at much larger parent bodies, maybe in the planetesimal size range (e.g. Lagrange et al. 2000). In this respect, even if there is a sharp outer edge for the parent body population, collisions would constantly produce very small grains that would be launched by radiation pressure onto eccentric or even unbound orbits, thus populating the region beyond the outer edge and erasing the appearance of a narrow ring over timescales which might be shorter than those for gravitational sculpting by a planet. This issue is a critical one, since these small grains dominate the total geometric cross section and thus the flux in scattered light (see the discussion in Thébault \& Augereau 2007).

One possible confining mechanism for the sharp outer edge is gas. For discs transiting between gas-dominated phase (protoplanetary discs) to dust-dominated phase (debris discs), narrow dust rings may arise (Klahr \& Lin 2005). Besla \& Wu (2007) further demonstrate that there exists an instability with which the residual gas collects grains of various sizes (even those subject to radiation pressure) into a narrow belt. However, this mechanism requires at least comparable amount of gas and dust. This may be difficult to justify for most debris discs, which are evolved systems where the amount of gas is probably too low to prevent the smallest grains to be launched onto very eccentric orbits smoothing out any sharp outer edge.

"Razor sharp" outer edges are thus very difficult to explain in the presence of this unavoidable outward launching of small grains. However, although no perfect abrupt outer edge has indeed been observed ${ }^{1}$ (unlike inner edges, which are in some cases, like Fomalhaut, almost razor sharp), a great variety of outer edge profiles does exist, from relatively smooth to very steep (see Table 1).

In this study, we address the issue of how these different profiles can be physically achieved: can this diversity be explained by the sole "natural" evolution of a collisional active disc steadily producing small, radiation-pressure affected grains, or is (are) additional mechanism(s) needed? We consider initial conditions which are a priori the most favourable for creating sharp edges, by assuming a population of large parent bodies confined within an annulus with an abrupt cutoff at its outer edge. How this initial confinement may have come about is itself an interesting question but is not the focus of the current paper (see however the discussion in Sect. 5.2). The outcome we consider as a reference for our investigation is the radial surface brightness (SB) profile in scattered light, since this is an observable which is reasonably well constrained for most imaged debris discs (either directly observed or obtained by de-projection). We consider the nominal case of a disc seen edge-on, but results can easily be extrapolated to face-on systems, since SB profiles for both orientations tend towards the same radial dependence far from the birth ring (given the same radial dust distribution).

In several previous studies, all addressing the specific $\beta$ Pic case (Lecavelier et al. 1996; Augereau et al. 2001; Thébault \& Augereau 2005), it has been argued that the "natural" SB profile outside the collisionaly active parent bodies belt, or "birth ring", falls off as $\sim r^{-5}$. This is based on the assumption that all particles produced in the birth ring have a size distribution which scales as $\mathrm{d} N / \mathrm{d} s \propto s^{-3.5}$, (as expected for an idealized infinite collisional cascade at equilibrium, see Dohnanyi 1969), down to the radiation blow-out limit $s=s_{0.5}$ (where the ratio of radiation pressure to gravity $\beta=0.5$ ). The smallest radiation pressureaffected grains, which dominate the light receiving area, are then diluted along their eccentric orbit. This geometrical spread results in $S B \propto r^{-5}$. However, Strubbe \& Chiang (2006) argued that, since high- $\beta$ grains spend a long time in the collisionaly inactive region beyond the birth ring, the $\mathrm{d} N / \mathrm{d} s \propto s^{-3.5}$ collisional equilibrium law should only apply to the small fraction $f$ of these grains which are present in the collisionaly active birth

\footnotetext{
${ }^{1}$ HD 141569A could be a possible exception, but this system is likely not a true debris disc, being significantly younger and gas rich (Jonkheid et al. 2006), but a member of the loosely defined "transition object" category.
} 
ring. This results in a $1 / f$ excess of the disc-integrated number of small grains, which in turn results in a flatter SB profile in $r^{-3.5}$. They applied their theory to the AU Mic disc ${ }^{2}$ and reproduced the observed SB profile, spectral energy distribution and disc colour. Strubbe \& Chiang (2006) further argued that the observed SB profile depends only weakly on the radial and size distributions of grains within the birth ring. The discs which exhibit a fall-off sharper than $r^{-3.5}$ are thus puzzling in the face of this theory.

The innovative model of Strubbe \& Chiang (2006) is build on analytical derivations and Monte-Carlo modeling which did not actually treat the collisional evolution of the system and relies on several simplifying assumptions. The main one is that the size distribution is fixed and is assumed to follow the idealized $\mathrm{d} N / \mathrm{d} s \propto s^{-3.5}$ scaling (corrected by the fraction $1 / f$ ), whereas several studies have shown that this law cannot hold in real systems (see Thébault \& Augereau 2007, and references therein) ${ }^{3}$. Another issue is that when evaluating collisional life-times, only the vertical velocity of the grains was taken into account, thus neglecting their radial movement which can be appreciable, if not dominant for the smallest grains. Finally, the specific dynamics of the small radiation-pressure-affected grains, in particular the fact that they suffer much more frequent collisions and at much higher velocities, is not taken into account.

\section{Our approach}

We re-address these issues using a numerical approach quantitatively following the collisional evolution of the full system. We start with a birth ring of parent bodies in a perfectly confined annulus and let it collisionally evolve. The temporal as well as spatial evolution of the size distribution are followed, taking into account the radial excursions of high- $\beta$ particles. As previously mentioned, we derive for each simulation the surface brightness profile in scattered light.

\section{1. numerical model}

We use a statistical particle-in-a-box model to follow the evolution in size and spatial distribution of a population of collisionally interacting bodies. This code has initially been developed, in its single-annulus version, for the study of the inner $\beta$ Pic disc (Thébault et al. 2003), and later upgraded to a multi-annulus version (i.e., with $1 \mathrm{D}$ radial resolution) for the study of collisional processes in extended debris discs (Thébault \& Augereau 2007). The detailed description of the code can be found in these two papers, and here we recall some of its main characteristics.

The entire system is spatially divided into $n_{\mathrm{a}}$ concentric radial annuli. Within each annulus of index $i a$, the solid body population is divided by size into $n_{\mathrm{s}}$ bins that cover a broad size range spanning from kilometre to micron. With a standard $\log (2)$ size increment, this requires $n_{\mathrm{s}} \sim 100$. Evolution of the particle number within one $(i a, i)$ bin ( $i$ being the size distribution index) is contributed by all destructive impacts between $(i a, i)$ objects and bodies from other $\left(i a^{\prime}, i^{\prime}\right)$ bins as well as all impacts between other size bins producing new $(i a, i)$ objects. Collision rates are estimated statistically. These rates, as well as collision outcomes,

\footnotetext{
$2 \mathrm{AU}$ Mic is an M star with weak radiation but where stellar wind is believed to act on small grains in an equivalent way as radiation pressure does around more massive A stars (e.g. Augereau \& Beust 2006).

3 The need for realistic size distributions departing from fixed power laws has been very recently emphasized by Fitzgerald et al. (2007) in their analysis of the AUMic data.
}

depend on average values of the encounter velocities $\Delta v_{\left(i a, i, i a^{\prime}, i^{\prime}\right)}$. For objects not subject to significant radiation pressure, relative velocities are obtained from the eccentricity and inclination distributions through the classical expression, valid for randomized orbits (e.g. Lissauer \& Stewart 1993):

$\langle\Delta v\rangle_{\left(i a, i, i^{\prime}\right)}=\left(\frac{5}{4}\left\langle e^{2}\right\rangle+\left\langle i^{2}\right\rangle\right)^{1 / 2}\left\langle v_{\mathrm{kep}(i a)}\right\rangle$

where $v_{\mathrm{kep}(i a)}$ is the Keplerian velocity at radial distance $r_{i a}$.

For the smallest particles, the effect of radiation pressure, which places objects on highly eccentric orbits, is taken into account. Inter annuli interactions, induced by the significant radial excursion of these bodies are considered, and $\Delta v_{\left(i a, i, i a^{\prime}, i^{\prime}\right)}$ are derived through separate deterministic $N$-body runs.

Collision outcomes are divided into 2 types, cratering and fragmentation, depending on the ratio between the specific impacting kinetic energy $E_{\mathrm{col}}$ and the specific shaterring energy $Q *$, which depends on object sizes and composition. In both regimes, the size distributions of the newly produced fragments are derived through detailed energy scaling prescriptions, which are presented at length in Thébault et al. (2003) and Thébault \& Augereau (2007). Possible reaccumulation onto the impacting objects is also accounted for.

\subsection{Set up}

Our numerical model requires the following inputs: the ring's average distance from the star, $r_{\mathrm{BR}}$, its radial width $\Delta r_{\mathrm{BR}}$, the average free eccentricities $\langle e\rangle$ and inclinations $\langle i\rangle$ of the parent bodies (non affected by radiation pressure) and the initial particles' size distribution and total mass. We chose to parameterize the latter by $M_{\text {dust }}$, the total mass of objects with sizes $s<s_{\mathrm{d}}=1 \mathrm{~cm}$, even though our simulations include bodies up to $10 \mathrm{~km}$, because the total "dust" mass is a parameter which can often be constrained from observations. We also assume equipartition between eccentricity and inclination, so that $\langle e\rangle=2\langle i\rangle$, at least for the parent bodies.

Due to the numerical cost of the detailed size distribution evolution procedure, $\langle e\rangle$ is assumed fixed, independent of position and time. The first independency is justified as we are considering a relatively narrow birth ring. The time independency is an acceptable simplification when considering that the eccentricity dispersion is imposed by the largest bodies present in the system (e.g. Quillen 2007b) and that, according to our current understanding of debris discs, i.e. systems in which the bulk of the planetesimal accretion process is already over, these bodies should be large planetary embryos that are too big and isolated to be significantly affected by collisional erosion over the $\sim 10^{7}-10^{8}$ yr timescale considered here (see, e.g. Wyatt et al. 2007; Löhne et al. 2008). This is also why these dynamicsimposing embryos are left out of the collisional cascade numerically studied here (for more on the subject, see Thébault \& Augereau 2007, as well as the discussion in Sect. 5.1.2).

For the sake of clarity, we consider a nominal case (Table 2) with set-up matching as closely as possible the $\beta$ Pictoris system, i.e. $M_{\text {dust }}=0.1 M_{\oplus}$ and $\langle e\rangle=0.1$ (e.g. Augereau et al. 2001). For the radiation pressure blow out size, we take $s_{0.5}=5 \mu \mathrm{m}$, the value derived for compact silicates around a $\beta$ Pic like A5V star. The system is divided into two radial zones:

- The parent body zone, or "birth ring", located between $r_{\text {in }}$ and $r_{\text {out }}$ with the center at $r_{\mathrm{BR}}$ and divided into 6 annuli, where we follow the collisional evolution of the whole solid 
Table 2. Nominal case set up. The fields marked by a * are explored as free parameters in the simulations.

\begin{tabular}{ll}
\hline \hline Radial extent of birth ring* & $80<r<120 \mathrm{AU}$ \\
Number and radial width of annuli* & $6 \times 6.66 \mathrm{AU}$ \\
Initial surface density within ring & uniform \\
Total "dust" mass $M_{\text {dust }}(s<1 \mathrm{~cm})^{*}$ & $0.1 M_{\oplus}$ \\
Stellar spectral type* $^{*}$ & A5V \\
Radiation Blow-out size* & $s_{0.5}=5 \mu \mathrm{m}$ \\
Size range modelled & $2 \mu \mathrm{m}<s<10 \mathrm{~km}$ \\
Number of size bins & 99 \\
Initial size distribution* & $\mathrm{d} N \propto s^{-3.5} \mathrm{~d} s$ \\
Dynamical excitation* & $\langle e\rangle_{0}=0.1=2\langle i\rangle_{0}$ \\
\hline
\end{tabular}

body population, from a maximum size $s_{\max }=10 \mathrm{~km}$ that sits in the planetesimal size range to a minimum size $s_{\min }=$ $2 \mu \mathrm{m}$ below the radiation blow-out limit. The initial size distribution in the entire range is assumed to follow the idealized collisional "equilibrium" distribution, $\mathrm{d} N(s)_{0} \propto s^{-3.5} \mathrm{~d} s$ (Dohnanyi 1969). Our runs show that this choice is not crucial: in the relevant dust-size range, the size distribution is quickly relaxed toward a new steady state with a profile that is independent of the initial choice and that deviates significantly from a Dohnanyi-like power law (see Sect. 4 and Fig. 9).

- The outer zone, which is devoid of particles at the beginning of the runs and gets progressively populated by small grains coming from the birth ring. Consequently, we only follow here grains with $s \leq s_{\max }^{*}$, where $s_{\max }^{*}$ roughly corresponds to the biggest grains able to leave the parent body region and is taken conservatively to have a radiation-to-gravity-ratio of $\beta_{\left(s_{\max }^{*}\right)}=0.1$. Spatially, this region is divided into 3 annuli just outside the main ring plus one additional, infinitely extended "buffer" annulus. Within the latter zone, no collisional evolution is modeled, and only the orbital evolution of the grains is considered: either escape of the system for unbound grains or progression to the apoastron and return to the inner annuli for the bound ones. The radial extent of the outer zone (not including the "buffer" annulus) is set to $\sim 4 r_{\mathrm{BR}}$, which is typically the extent of the "outer" region considered for the 2 most famous birth-ring/outer-zone systems, i.e., $\beta$ Pic and AU Mic (e.g. Augereau et al. 2001; Strubbe \& Chiang 2006).

The radial surface brightness profile in scattered light is then synthetically computed using the dust size and radial distributions, assuming an $r^{-2}$ dilution of the stellar flux and isotropic scattering (although different scattering properties are also explored).

We explore around the nominal set-up (see Table 2), especially for the two fundamental parameters which are $M_{\text {dust }}$ and the dynamical excitation of the system (as parameterized by $\langle e\rangle$ ).

\section{Numerical results}

\section{1. nominal case: smooth edge and $r^{-3.5}$ profile}

Figure 1 presents results obtained for our $\beta$ Pic-like nominal case. As can be clearly seen, the system rapidly $\left(\sim 10^{5} \mathrm{yr}\right)$ reaches a steady state after which the SB profile no longer evolves significantly. We stop the integration at $10^{7} \mathrm{yr}$. This $\sim 10^{5} \mathrm{yr}$ timescale is the time it takes for the small grains that fill the outer radial zone to reach an equilibrium between collisional production and destruction. A direct consequence of this fast

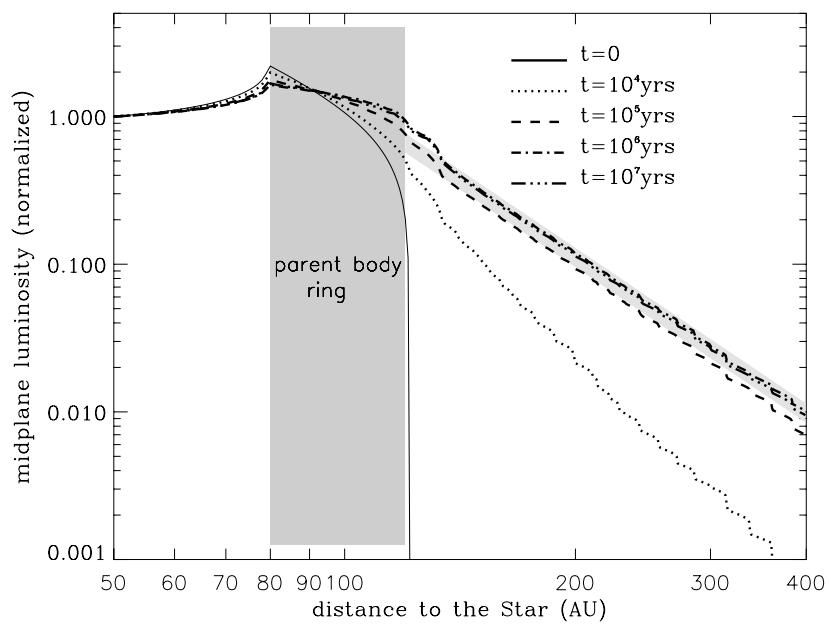

Fig. 1. Evolution of the midplane luminosity profile in scattered light. Nominal case: parent body ring [80,120] $\mathrm{AU}, M_{\text {dust }}=0.1 M_{\oplus},\langle e\rangle=$ 0.1 . Each midplane luminosity has been renormalized to its value at $50 \mathrm{AU}$. The dark grey area represents the parent body region where all mass is initially located. The narrow light grey area represents a $r^{-3.5}$ slope reconstructed backwards from the final luminosity value at 400 AU with a $\pm 15 \%$ width.

evolution is that the initial sharp outer edge of the parent body region is quickly smoothed out. Once the steady state is reached, the profile in the $r>r_{\text {out }}$ region lies very close to $S B(r) \propto r^{-3.5}$.

When looking at the respective contributions from different dust populations to the total profile, it appears that the scattered flux is, in the $120-400$ AU region, dominated by high- $\beta$ grains in the $0.25<\beta<0.4$ range (Fig. 2). These grains have orbital eccentricities in the $0.33-0.75$ range and apoastron in the 240-700 AU region and thus spend a large fraction of their orbits in the domain located between $r_{\text {out }}$ and $4 r_{\mathrm{BR}}$. Grains with even higher $\beta$ (close to 0.5 ) only weakly contribute to the flux in the $r_{\text {out }}$ to $4 r_{\mathrm{BR}}$ region because they have orbits whose apoastron is often at several $1000 \mathrm{AU}$ and will thus spend most of their time outside the $\leq 400$ AU region considered here.

For a case with a much less massive $\left(M_{\text {dust }}=0.001 M_{\oplus}\right)$ disc, we see that the system reaches a similar outer SB profile $\left(S B \propto r^{-3.5}\right.$ outside $\left.r_{\text {out }}\right)$ but with a much longer timescale: a few $10^{7} \mathrm{yr}$ instead of $\sim 10^{5} \mathrm{yr}$ in the nominal run (Fig. 3). Similar SB profile is also obtained for a higher mass run $\left(M_{\text {dust }}=1 M_{\oplus}\right)$. Once again, the only difference with the nominal case is the pace at which the steady-state is reached (almost $10^{4} \mathrm{yr}$ here).

We further explore the parameter dependences by running a series of simulations varying the width of the birth ring and the dynamical excitation of the system. We also investigate the importance of the scattering function assumption by exploring anisotropic cases, assuming a Henyey \& Greenstein (1941) phase function and changing the asymmetry factor $|g|$. The results for all these runs are summarized in Table 3, showing the 2 main outcomes of interest for the present problem: the luminosity drop at the outer edge of the ring and the average slope of the luminosity profile in the $1.5 r_{\mathrm{BR}}-4 r_{\mathrm{BR}}$ region. The nominal results are robust: the luminosity drop at the ring's outer edge is always comprised between 2 and 3 , whereas for the slope we get $\alpha=-3.51 \pm 0.03$. The only way to reach a somehow steeper slope is to assume a strong anisotropy of the scattering function. But even for a rather extreme $g=0.8$ case, $\alpha \sim-3.98$. 


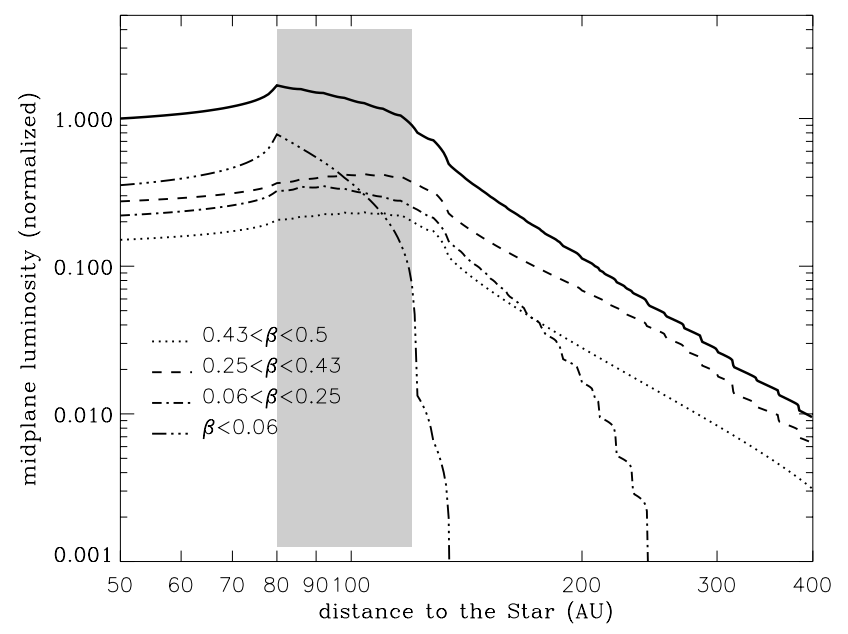

Fig. 2. Nominal case at $t=10^{7} \mathrm{yr}$. Respective contributions of different grain populations (parameterized by their $\beta$ value) to the total scattered flux, as functions of the radial distance.

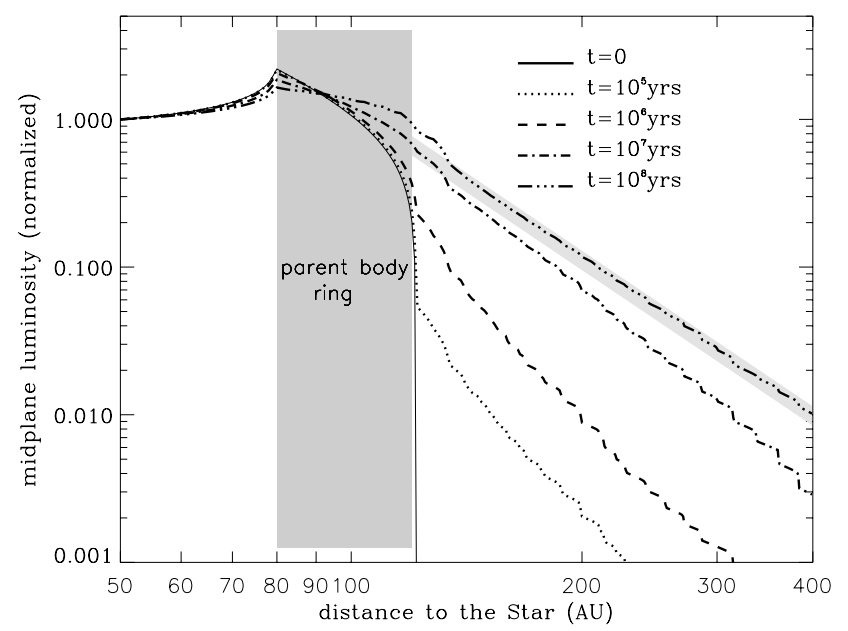

Fig. 3. Same as Fig. 1, but with $M_{\text {dust }}=0.001 M_{\oplus}$, all other parameters being the same. Note that the final timescale is here $t=10^{8} \mathrm{yr}$.

\section{2. "extreme cases" producing different profiles}

\subsection{1. very massive disc}

In order to push our parameter exploration to the limits, we keep increasing the total initial dust mass $M_{\text {dust }}$ until we observe a departure from the nominal case SB profile. Such a departure is observed for $M_{\text {dust }}=10 M_{\oplus}$ (Fig. 4). This extremely massive case distinguishes itself from the others by the fact that a sharp luminosity drop is maintained at the outer edge for $\sim 10^{6} \mathrm{yr}$. What keeps the outer regions at a very low luminosity is the fact that the parent body ring is so densely populated that grains pushed outward by radiation pressure cannot freely escape it without experiencing a collision. This can be illustrated by looking at a simplified parameter, i.e. the geometrical radial optical depth, defined (at a given distance from the star $r_{0}$ ) by

$\tau_{\mathrm{rad}}\left(r_{0}\right)=\sum_{i a=i 0}^{n_{\mathrm{a}}} \frac{\int \mathrm{d} N_{i a} / \mathrm{d} s(r) \pi s^{2} \mathrm{~d} s}{2 \pi r H}$

where $\mathrm{d} N_{i a} / \mathrm{d} s(r)$ is the differential number of $s$-sized particles in a radial annulus of index $i a$ centered at radial distance $r, H$
Table 3. Results obtained for different values of the system's dynamical excitation $\langle e\rangle(=2\langle i\rangle)$ and width of the birth ring $\Delta r_{\mathrm{BR}}{ }^{a}$. Also shown are results for the nominal run but with the SB computed using a different scattering phase function (see text for details).

\begin{tabular}{lcc}
\hline \hline Run & $\begin{array}{c}\text { Outer edge sharpness }^{b} \\
S B\left(r_{\text {out }}\right) / S B\left(r_{\text {out }+10}\right)\end{array}$ & $\begin{array}{c}\text { SB profile }^{c} \\
\text { slope } \alpha\end{array}$ \\
\hline nominal case & 2.50 & -3.51 \\
$\langle e\rangle=0.2$ & 2.44 & -3.48 \\
$\langle e\rangle=0.035$ & 2.78 & -3.52 \\
$\langle e\rangle=0.01$ & 3.33 & -3.49 \\
$\Delta r_{\mathrm{BR}}=20 \mathrm{AU}$ & 2.05 & -3.51 \\
$\Delta r_{\mathrm{BR}}=10 \mathrm{AU}$ & 1.96 & -3.54 \\
\hline scattering anisotropy $g=0.5$ & 2.22 & -3.68 \\
scattering anisotropy $g=0.8$ & 2.22 & -3.98 \\
\hline
\end{tabular}

${ }^{a}$ When varying its outer edge $r_{\text {out }}$, its inner edge being fixed at $80 \mathrm{AU}$. ${ }^{b}$ Luminosity drop at the outer edge of the ring, as measured by the flux ratio between the outer edge of the birth-ring and $10 \mathrm{AU}$ beyond it.

${ }^{c}$ Average value in the $1.5 r_{\mathrm{BR}}-4 r_{\mathrm{BR}}$ region.

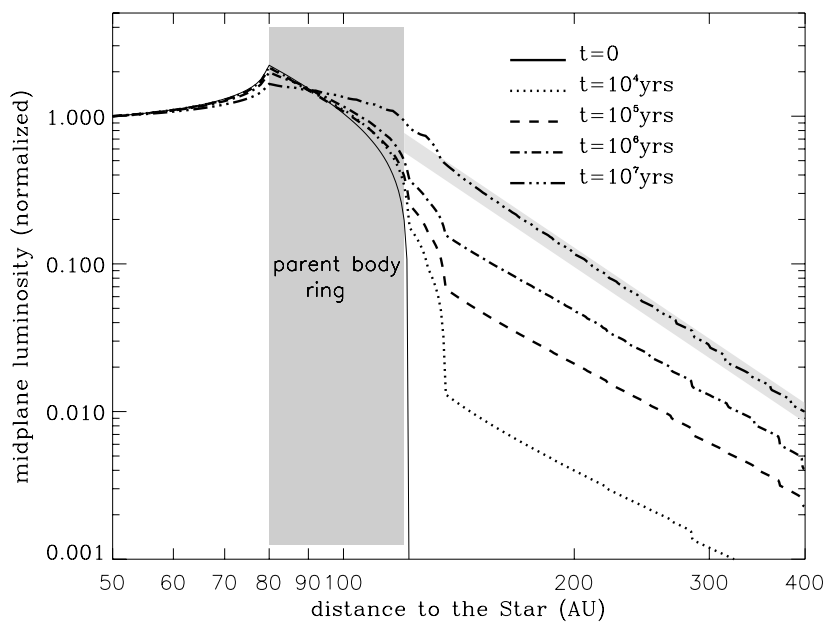

Fig. 4. Same as Fig. 1, but with a very high dust mass of $M_{\text {dust }}=10 M_{\oplus}$.

is the vertical height of the disc at that distance and $i 0$ is the annulus index corresponding to the radial distance $r_{0}$. $\tau_{\text {rad }}$ is of course simply the optical thickness of the disc to stellar photons. Since particle orbits are never straight radial lines, this quantity is only a first approximation of their real in-plane (or horizontal) collisional probability. However, for the smallest grains this is a relatively good first-order approximation (see the more thorough discussion on horizontal and vertical collision probabilities in Sect. 5.1).

Figure 5 shows that $\tau_{\text {rad }} \geq 1$ over most of the source ring ${ }^{4}$ in the early epoch, so that few grains can escape the birth ring without colliding with another grain, hence the density (and luminosity) depletion in the $r>r_{\text {out }}$ regions. However, this radial optical depth steadily decreases over time, due to rapid mass erosion by energetic collisions within the birth ring. It drops below unity after $\sim 10^{5} \mathrm{yr}$, and is $\sim 0.6$ by the time $\left(\sim 10^{6} \mathrm{yr}\right)$ the sharp outer edge is smoothed out (see Fig. 4). After that, the system behaves like the nominal case. We re-examine this radially optically thick case in more detail in Sect. 5.1.

\footnotetext{
${ }^{4}$ When this is the case, one should also include the attenuation of stellar light over the disc to be self-consistent. However, we believe doing so would not qualitatively alter our conclusion.
} 


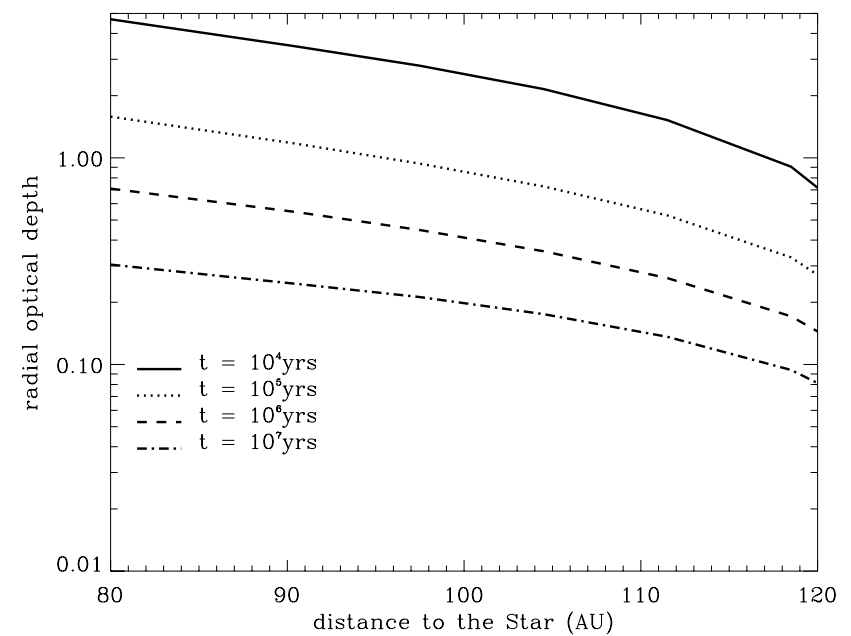

Fig. 5. Evolution with time of the radial optical depth (defined as in Eq. (2)) for the very high dust-mass case $M_{\text {dust }}=10 M_{\oplus}$.

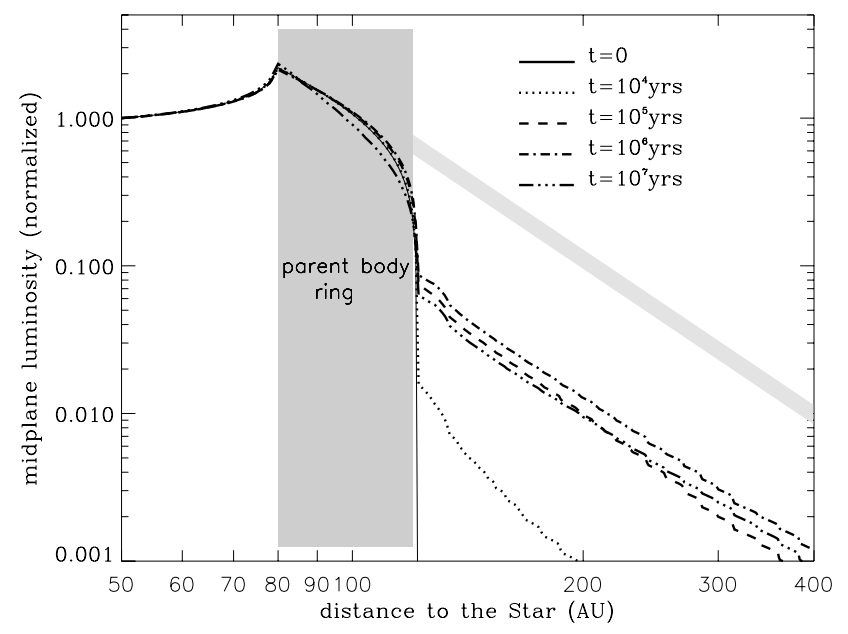

Fig. 6. Same as Fig. 1, but for a dynamically "very cold" system with $\langle e\rangle=0.001$.

\subsection{2. dynamically cold system}

Another way of obtaining a departure from the standard SB profile is to decrease the dynamical excitation of the system to a very low value, typically $\langle e\rangle \leq 0.01$. Figure 6 shows the results for the case $\langle e\rangle=0.001(=2\langle i\rangle)$, where a sharp outer edge is maintained outside the birth ring for the entire duration of the simulation $\left(10^{7} \mathrm{yr}\right)$.

The abrupt luminosity fall-off at the outer edge is here due to a global depletion of small grains directly resulting from an imbalance between the collisional production and destruction rates of small and large particles in such a dynamically cold disc. This can be understood in the following way:

For a given dust mass, a lower dynamical excitation does not change the collision rate between large objects not affected by radiation pressure. Indeed, because of the equipartition $\langle e\rangle=$ $2\langle i\rangle$ the decrease in $\langle\Delta v\rangle$ is exactly compensated by the increase of the particle number density due to the reduced thickness. However, the lower $\langle\Delta v\rangle$ values mean that collisions will be less destructive and produce less smaller fragments. This means that the rate at which small grains (the ones significantly affected by radiation pressure) are produced is significantly reduced. On the contrary, the rate at which these small grains are destroyed is higher. Indeed, collisions velocities for impacts involving them

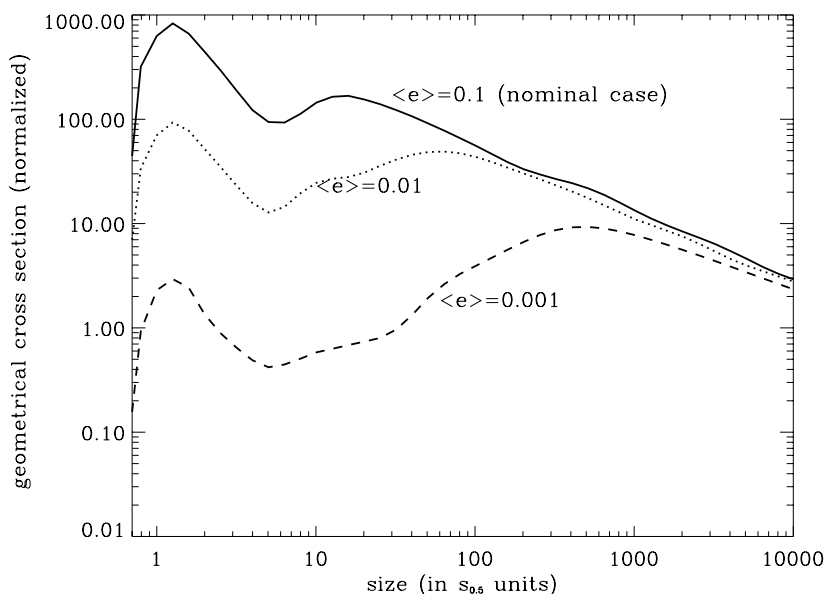

Fig. 7. Geometrical cross section per logarithmic size range, integrated only for grains inside the birth ring, for systems with different dynamical excitation. These curves at obtained at the end of the integration $\left(10^{7} \mathrm{yr}\right)$ and are normalized to have the same value at the large grain size.

are not significantly reduced by the small $\langle e\rangle$ for parent bodies, since small grain dynamics is predominantly imposed by radiation pressure. Furthermore, the rate at which such impacts occur is increased, compared to the nominal case, because of the increased radial optical depth (see Sect. 5.1.2 for a more detailed analysis).

As $\langle e\rangle$ decreases, this imbalance becomes more severe and the depletion of the smallest grains is more acute. This is illustrated in Fig. 7 displaying, for different values of the system's dynamical excitation, the respective contributions of different grain sizes to the total geometrical cross section $\sigma$. For the nominal case $(\langle e\rangle=0.1)$, we obtain the standard result that $\sigma$ is dominated by the smallest grains close to the cut-off size. As $\langle e\rangle$ gets smaller, however, the contribution of these smaller grains progressively decreases. Below the limiting value $\langle e\rangle \sim 0.01$, this effect is so pronounced that the system's optical depth is no longer dominated by the smallest grains, but by much bigger particles in the 100-1000s $s_{0.5}$ range. These particles have their orbits largely confined within the birth ring. This explains why a sharp luminosity decrease is observed at the outer edge of the birth ring.

Note that contrary to the very high mass case, the sharp outer edge does not smooth out with time but persist throughout the $10^{7} \mathrm{yr}$ of the simulation.

\section{Analytical derivation: the "universal" $r^{-3.5}$ profile}

Our numerical exploration has shown that the $r^{-3.5}$ surface brightness profile beyond the outer edge seems to be the most generic outcome for a collisional ring system under the action of stellar radiation pressure. We reinvestigate this issue from an analytical point of view and derive simplified formulae confirming this result. We take here as a basis the analytical approach of Strubbe \& Chiang (2006) and extend it to more general cases regarding grain size and spatial distributions.

We firstly assume that, within the birth ring, particles follow a power-law size distribution of index $q$ (instead of fixing $q=$ -3.5 for a Dohnanyi equilibrium):

$\mathrm{d} N_{\mathrm{BR}} \propto s^{q} \mathrm{~d} s$.

Since these grains spend most of their orbits in the empty region outside the birth ring, their total number integrated over the 
whole system $\left(N_{\text {tot }}\right)$ will be boosted by a factor $1 / f(e)$, where $f(e)$ is the fraction of an orbital period a body of eccentricity $e \sim \beta /(1-\beta)$ spends within the birth ring (Strubbe \& Chiang 2006), so that

$\mathrm{d} N_{\text {tot }}=\frac{1}{f(e)} \mathrm{d} N_{\mathrm{BR}} \propto \frac{1}{f(e)} s^{q} \mathrm{~d} s$.

We also follow Strubbe \& Chiang (2006) in making the simplifying but reasonable assumption that all high- $\beta$ grains are on average mostly seen near their apoastron. Thus, at each given distance $r$ from the star, the optical depth is dominated by particles of size

$s_{\mathrm{dom}}(r)=\frac{1}{1-\frac{r_{\mathrm{BR}}}{r}} s_{0.5}$.

Since we are interested in the region $r \leq 4 r_{\mathrm{BR}}$, only grains with their apoastron

$a(1+e)=\frac{1-\beta}{1-2 \beta}\left(1+\frac{\beta}{1-\beta}\right) r_{\mathrm{BR}}=\frac{r_{\mathrm{BR}}}{1-2 \beta}<4 r_{\mathrm{BR}}$

are important. This corresponds to $\beta<0.4$ and thus $e<0.67$. Note that, in their analytical derivation (this assumption was relaxed in their Monte-Carlo model), Strubbe \& Chiang (2006) only considered extremely eccentric $e \sim 1$ grains, which spend most of their orbits outside the considered $\leq 4 r_{\mathrm{BR}}$ region.

To estimate the enhancement ratio $1 / f(e)$, we do not adopt the asymptotic expression (valid for $e \sim 1) f(e) \propto(1-e)^{1.5}$ taken by Strubbe \& Chiang (2006) but instead derive this value from Kepler's equation for a typical particle produced at the middle of the birth ring:

$f(e) \propto\left(E_{2}-E_{1}\right)-e\left(\sin \left(E_{2}\right)-\sin \left(E_{1}\right)\right)$

where

$E_{1}=0 \quad$ and $\quad E_{2}=a \cos \left[\frac{1}{e}\left(1-\frac{r_{\mathrm{BR}}+\Delta r_{\mathrm{BR}}}{r_{\mathrm{BR}}}\right)\right]$.

The vertical geometrical optical depth then scales with radius as

$$
\begin{aligned}
\tau_{\perp}(r) & \propto \frac{1}{r} \frac{\mathrm{d} N_{\mathrm{tot}}\left(s_{\mathrm{dom}}\right)}{\mathrm{d} r} s_{\mathrm{dom}}^{2}(r) \\
& \propto \frac{1}{f(e)} \frac{1}{r} \frac{\mathrm{d} N_{\mathrm{BR}}\left(s_{\mathrm{dom}}\right)}{\mathrm{d} r} s_{\mathrm{dom}}^{2}(r) .
\end{aligned}
$$

Using the radial dependence of $s_{\text {dom }}$ given by Eq. (5), we obtain $\frac{\mathrm{d} N_{\mathrm{BR}}\left(s_{\mathrm{dom}}\right)}{\mathrm{d} r} \propto\left(1-\frac{r_{\mathrm{BR}}}{r}\right)^{-q-2} \frac{r_{\mathrm{BR}}}{r^{2}}$.

Inserting Eqs. (4), (5) and (10) into Eq. (9) leads to

$\tau_{\perp}(r) \propto \frac{1}{f(e)}\left(1-\frac{r_{\mathrm{BR}}}{r}\right)^{-q-4} \frac{r_{\mathrm{BR}}}{r^{3}}$

where $f(e)$ is given by solving Eq. (7).

As a test, we first consider the same fiducial case as Strubbe $\&$ Chiang (2006), i.e. the Dohnanyi $q=-3.5$ value. The radial profile of $\tau_{\perp}(r)$ obtained this way scales as $r^{-1.5}$ (Fig. 8). Departures from this slope are relatively limited, even in the $1-1.5 r_{\mathrm{BR}}$ region, where $\tau_{\perp}(r)$ has a $\sim r^{-1.75}$ dependence. If we apply the usual rule of thumb that for an optical depth profile $\tau_{\perp}(r) \propto r^{\alpha}$, the midplane SB scales as $r^{\alpha-2}$ (e.g. Nakano 1990) ${ }^{5}$,

5 This assumes grey scattering and that the disc's vertical thickness scales as $H \propto r$.

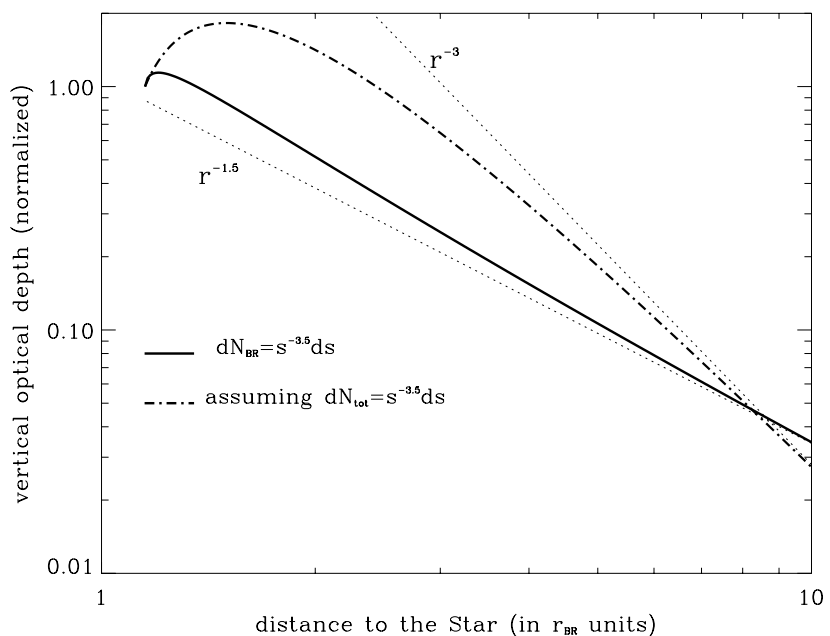

Fig. 8. Radial profile of the vertical optical depth, obtained with our analytical fit of Eq. (11), assuming $q=-3.5$ (with a ring width $\Delta r_{\mathrm{BR}}=$ $\left.0.1 r_{\mathrm{BR}}\right)$. As a comparison, we also plot the profile obtained with the (incorrect) assumption that the size distribution is $\mathrm{d} N_{\text {tot }} \propto s^{-3.5} \mathrm{~d} s$ in the whole system.

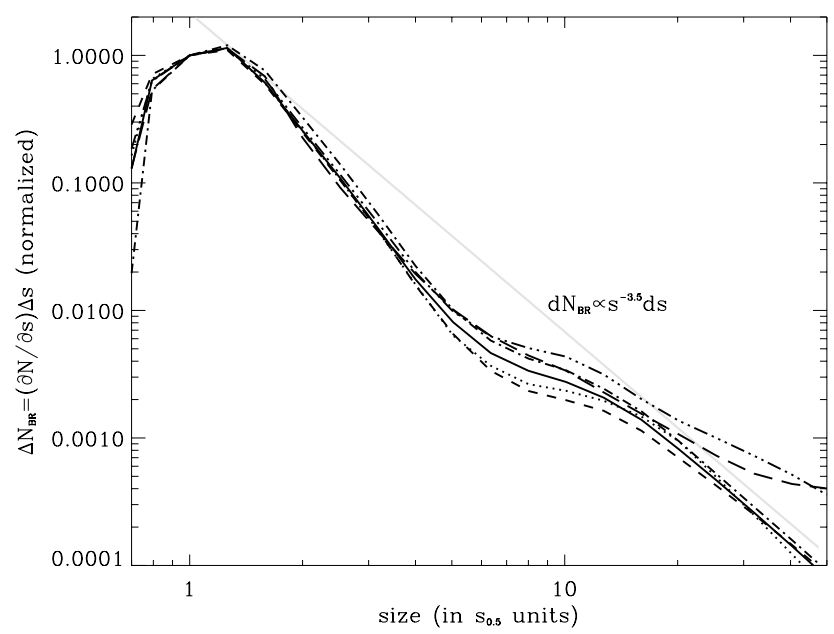

Fig. 9. Grain size distribution inside the birth ring $\left(\mathrm{d} N_{\mathrm{BR}} / \mathrm{d} s\right)$, for all cases presented in Sect. 3. The size range here covers that of grains dominating the optical depth in the outer region. In order to facilitate comparison, all curves have been renormalized to their peak value at $s=1.2 s_{0.5}$. The thick grey line shows the theoretical Dohnanyi profile $\mathrm{d} N_{\mathrm{BR}} \propto s^{-3.5} \mathrm{~d} s$.

we obtain $S B(r) \propto r^{-3.5}$ in most of the outer region, confirming the result of Strubbe \& Chiang (2006). For comparison, we also plot on the graph the $\tau_{\perp}(r)$ profile derived when (incorrectly) assuming that the Dohnanyi law applies to the entire system, i.e., $\mathrm{d} N_{\text {tot }} \propto s^{-3.5} \mathrm{~d} s$ (as was done in Lecavelier et al. 1996; Augereau et al. 2001; Thébault \& Augereau 2005). Not surprisingly, we recover the asymptotic dependence $\tau_{\perp}(r) \propto r^{-3}$, corresponding to the $S B(r) \propto r^{-5}$ slope derived in these past studies.

However, as has been often pointed out (e.g. Thébault et al. 2003), size distributions follow a power law with index $q=-3.5$ only in unrealistically idealized systems, with an infinite size range and no size dependence for collisional processes. In our simulations, the steady state size distribution within the birth ring significantly departs from this value (see Fig. 9). This departure from the Dohnanyi law, especially in the crucial size domain of the smallest grains, is a well known result which has been obtained and discussed in several previous studies (e.g. 


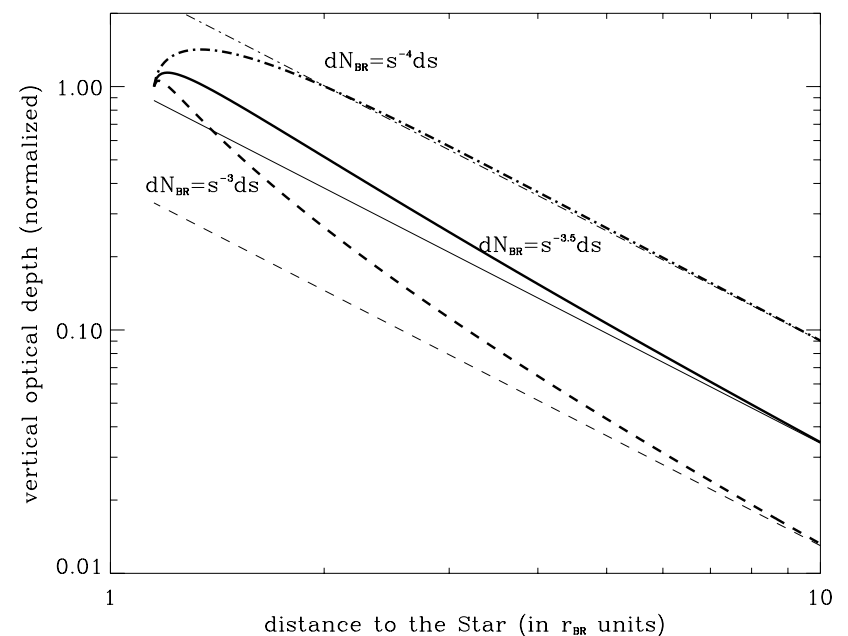

Fig. 10. Same as Fig. 8 but for different size distributions index $q$. All size distributions lead to $\tau_{\perp} \propto r^{-1.5}$ at large distances, but steeper size distributions approaching this asymptote earlier.

Campo Bagatin et al. 1994; Thébault et al. 2003; Krivov et al. 2006; Thébault \& Augereau 2007; Löhne et al. 2008). The main reason for this behaviour is that radiation pressure introduces a natural minimum cut-off $s_{0.5}$ in the size distribution, so that bodies of size $s_{1}$ just above $s_{0.5}$ are overabundant because of the lack of small $s<s_{0.5}$ impactors that could destroy them. Subsequently, larger bodies, of size $s_{2}$, which can be destroyed by impacts with $s_{1}$ ones, are underabundant, which in turn leads to an overabundance of objects of size $s_{3}>s_{2}$, etc. The resulting size distribution displays a pronounced wavy-pattern observable also in Fig. 9. For the size range which is here of special interest, i.e. grains in the $0.15<\beta<0.4\left(1.25 s_{0.5}<s<3.5 s_{0.5}\right)$ range, the distribution is steeper than a Dohnanyi one and is close to $q \sim-4$. Interestingly, putting this value into Eq. (11) leads to a $\tau_{\perp}(r)$ profile that is again well (in fact, better) approximated by the $r^{-1.5}$ slope (Fig. 10). This is because, in Eq. (11), the $r$ dependence arising from the size distribution term $\left(1-r_{\mathrm{BR}} / r\right)^{-q-4}$ is swarmed by that from the geometric term $\left(r_{\mathrm{BR}} /\left(f(e) \times r^{3}\right)\right.$ at large $r$. Only the rapidity at which the $r^{-1.5}$ asymptotic behaviour is reached depends on the index $q$ (Fig. 10).

\section{Discussion}

\subsection{How to escape the universal $r^{-3.5}$ profile}

Our numerical explorations show that there are only 2 ways not to end up with the standard result with no sharp edge and $S B \propto r^{-3.5}$ : a very massive, radially optically thick disc or a dynamically cold system. For these 2 "extreme" cases, the analytical derivation of the previous section becomes invalid. Although the reasons why the analytical study no longer holds are different for each case, they are nevertheless for both cases distinct consequences of the same crucial characteristics of the system's dynamics when pushed to its limits: the strong imbalance between the collision rates and velocities of large and small particles.

Therefore, before discussing these 2 cases in more detail, let us present the mechanisms at play behind this large/small particle dichotomy.

For large bodies, we have seen that collision velocities can be derived using the standard expression for their dynamical rms excitation (Eq. (1)). Since for these objects equipartition between in-plane and off-plane motions results in a fixed $\langle e\rangle /\langle i\rangle$ ratio, it follows that collision velocities are directly proportional

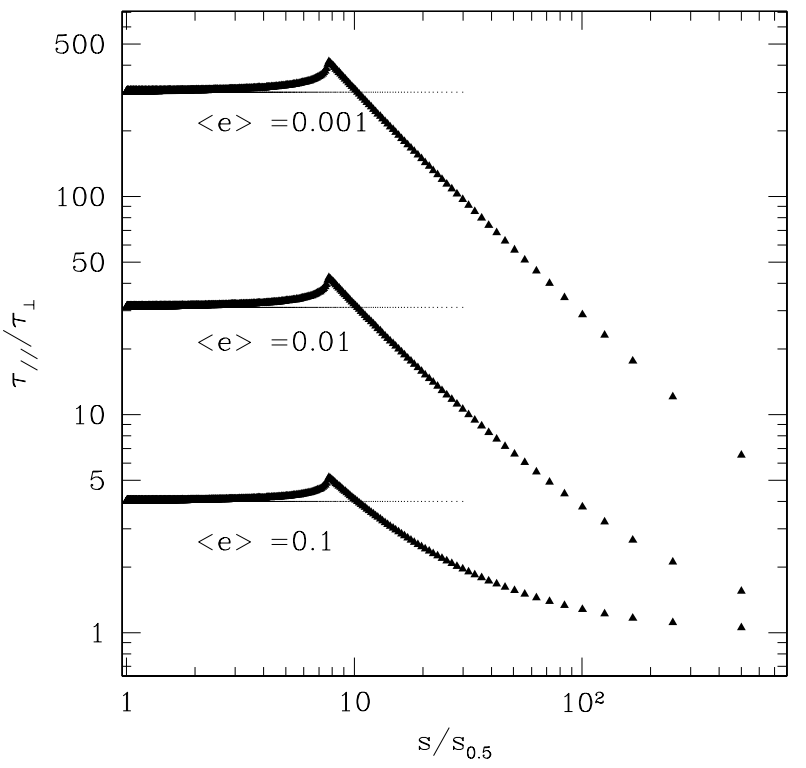

Fig. 11. Ratios between the horizontal and vertical optical depth plotted as a function of grain size for different values of dynamical excitation. The straight lines associated with each case mark the values of $\tau_{\text {rad }} / \tau_{\perp}$ (see Eq. (2)). The grains are assumed to be launched with local Keplerian circular velocity at the centre of the ring. The fractional ring width $\Delta r_{\mathrm{BR}} / r_{\mathrm{BR}}$ takes the nominal value of 0.3 . Decreasing this value will move the peaks towards the right.

to the system's inclination whereas collision rates are independent of $\langle i\rangle$. That is to say that the collisional optical depth is directly proportional to the vertical optical depth $\tau_{\perp}$ (which does not vary with $\langle i\rangle)$. This is no longer the case for small grains pushed on eccentric orbits by radiation pressure, which can sample the whole radial extent of the birth ring. Radiation pressure induced eccentricities are equal to

$e_{\beta}=\left(1-\frac{(1-2 a \beta / r)\left(1-\left\langle e^{2}\right\rangle\right)}{(1-\beta)^{2}}\right)^{1 / 2}$,

where $a$ is the semi-major axis of the parent body producing the small grain, and $r$ the radial location of its release. For these objects, in-plane motions become much more important than vertical ones. This can be quantitatively estimated by computing the in-plane collisional optical depth $\tau_{/ /}$. This quantity is obtained by integrating, for a given particle, over distances that are travelled by this grain relative to its background,

$\tau_{/ /}=\oint\left|\boldsymbol{v}-\boldsymbol{v}_{0}\right| \mathrm{d} t n \sigma$

where $\boldsymbol{v}$ is the grain velocity, $\boldsymbol{v}_{0}$ the mean velocity of particles in the region, which we approximate to the local circular Keplerian velocity. $n$ and $\sigma$ are the local number density and grain cross section, and their product is related to the vertical optical depth by $\tau_{\perp}=2 H n \sigma$. The integration $(\oint)$ is over the whole orbit and $n \sigma$ is non-zero only inside the birth ring. For a given particle $\tau_{/ /}$depends on 2 main parameters: its orbital eccentricity and, for the smallest grains, the birth ring's radial extent (since this is the only region where collisions can occur). For large objects, equipartition between $\langle e\rangle$ and $\langle i\rangle$ means that $\tau_{/ /}$is simply equal to $\tau_{\perp}$ (see Fig. 11), which is another way to say that impacts in the in-plane and vertical directions are equally important. As grains get smaller, however, the ratio $\tau_{/ /} / \tau_{\perp}$ increases, reaching a maximum when the grain's orbit has the same radial extent as 
the birth ring, hence the peak at $s \sim 7 s_{0.5}$ for the nominal birth ring considered in Fig. 11.

The imbalance between small and large grains collision rates is directly proportional to $e_{\beta} /\langle e\rangle$. Since $e_{\beta}$ only weakly depends on $\langle e\rangle$ (Eq. (12)), this means that the imbalance increases for dynamically cold systems, as clearly appears in Fig. 11.

\subsubsection{Radially optically thick disc}

In Sect. 3.2.1 we identified one consequence of the boosted collision rates of small particles, which is that, for very high $M_{\text {dust }}$ values, high- $\beta$ particles have a significant chance to experience a collision before escaping the birth ring. In this situation, the analytical derivation of the previous section is invalid because it implicitly assumes that all high- $\beta$ grains produced in the birth ring eventually reach their apoastron far in the outer regions.

To characterize this collisional optically thick case, we used in Sect. 3.2.1 the parameter $\tau_{\text {rad }}$, as defined in Eq. (2), and empirically found that the limiting $\tau_{\text {rad }}$ value is around 0.6 . The validity of the $\tau_{\text {rad }}$ parameterization is confirmed by Fig. 11, which shows that this simplified parameter is actually a good approximation of the "real" in-plane (or horizontal) collisional optical depth $\tau_{/ /}$for small grains close to the $s_{0.5}$ limit. We thus keep this easily defined parameter (as compared to $\tau_{/ /}$) as a good approximate limiting criterion. Writing as a first approximation that

$\tau_{\mathrm{rad}} \sim \frac{\Delta r_{\mathrm{BR}}}{r_{\mathrm{BR}}} \frac{1}{\langle e\rangle}\left\langle\tau_{\perp}\right\rangle_{\mathrm{BR}}$,

we see that there are two ways of reaching high $\tau_{\text {rad }}$ values: either by increasing the total dust mass of the system (as parameterized by $\tau_{\perp}$ or $M_{\text {dust }}$ ) or by decreasing its dynamical excitation.

We have seen in our numerical exploration that only playing on the $M_{\text {dust }}$ (or $\tau_{\perp}$ ) parameter requires to reach values of the order of $10 M_{\oplus}$ for our nominal case of a disc with $\langle e\rangle=0.1$ and $\Delta r_{\mathrm{BR}} / r_{\mathrm{BR}}=0.3$. However, our simulations show that global collisional erosion in such a high-mass system is so intense that the $\tau_{\text {rad }} \geq 0.6$ regime can only be maintained for $\sim 10^{6} \mathrm{yr}$ before the birth ring becomes radially transparent for high- $\beta$ grains, at which point the sharp outer edge vanishes (see Fig. 4). One might argue that the largest bodies in our simulation are in the $10 \mathrm{~km}$ range and thus can be quickly eroded in this high density case, while realistic systems may have a large mass reservoir contained in larger bodies and could sustain a longer period of extreme dustiness. However, in this case the total mass of solids in the system, which is already $M_{\text {tot }} \sim 10^{4} M_{\oplus}$ in our high-mass run with $s_{\max }=10 \mathrm{~km}$, would reach unrealistically high values. Another strong argument for ruling out this high-mass case is that the presence of $10 M_{\oplus}$ of dust is not supported by observations. Indeed, no debris disc around a main sequence star seems to contain more than $\sim 1 M_{\oplus}$ of dust (Greaves 2005), or has a vertical optical depth exceeding $\sim 10^{-2}$ (Kalas 2005).

The other alternative for reaching high $\tau_{\text {rad }}$ values, i.e. reducing the dynamical excitation, would require, for a typical $M_{\text {dust }}=0.1 M_{\oplus}$ disc, $\langle e\rangle$ values as low as $\sim 0.001$. However, for such low values of the excitation, we have seen that the system's collisional evolution and size distribution is already in the qualitatively different "dynamically cold" regime, where small particles cease to dominate the optical depth (see next section).

\subsubsection{Dynamically cold disc: depletion of small grains}

This dynamically cold mode, with typically $\langle e\rangle\langle 0.01$, is in fact the second way to maintain a sharp outer edge (see Sect. 3.2.2).
In this case, the imbalance between small and large grains' collisional behaviour gets very pronounced (as it appears clearly in Fig. 11), up to a point where it causes a depletion of small high$\beta$ grains. We have seen in Sect. 3.2.2 that this is because small grains are predominantly created by impacts involving large objects, which get less efficient for low $\langle e\rangle$, but predominantly destroyed by collisions involving themselves, whose efficiency (imposed by radiation pressure-induced motions) only weakly varies with $\langle e\rangle$ of the parent bodies and whose rate strongly increases for decreasing $\langle e\rangle$. As a consequence, contrary to the nominal case the system's optical depth and luminosity are dominated by larger $\sim 100 s_{0.5}$ grains that do not leave the birth ring, hence the sharp, and long-lived drop at its outer edge ${ }^{6}$.

However, we are here confronted with two issues.

The first one regards the total brightness of such discs. Indeed, a disc deprived from its smallest grain population (in the $\leq 100 s_{0.5} \sim 0.5 \mathrm{~mm}$ range) should appear much dimmer than a disc in which these grains dominate the light scattering area. From Fig. 7, we see that, for the same total mass of dust (always contained in the biggest grains), a small-grain-poor system with $\langle e\rangle \leq 0.01$ is at least 10 times less luminous than the nominal case. It follows that, to reach the same fractional luminosity (assuming it is proportional to the optical depth), the dynamically cold system has to be at least 10 times more massive. This has no direct implications on observed dust mass estimates, which are usually derived from sub-mm or millimetre observations in thermal emission, but should affect the correlation between $M_{\text {dust }}$ and total fractional luminosities $f_{L}$. In this respect, dynamically colder (or thinner) discs should have higher $M_{\text {dust }} / f_{L}$ ratios. However, the uncertainties affecting both parameters' estimates are probably high enough to accommodate a factor 10 uncertainty in their ratio (see for example the attempt at connecting these two quantifies for the specific case of AU Mic performed by Augereau \& Beust 2006), so that our result is here probably not very constraining.

The second and probably crucial issue is how likely it is to find such dynamically cold discs, with $\langle e\rangle$ and $\langle i\rangle$ lower than $\simeq 0.01$. As noted by Thébault \& Augereau (2007): "the only observational constraint [on the disc's dynamical excitation] comes from measuring the disc's vertical thickness and deriving estimates of orbital inclinations, but such constraints are scarce". Edge-on discs represent the most favourable cases since $H / r$ can be directly measured. However, even for the two most studied discs, only partial information is available. Krist et al. (2005) find $H / r \leq 0.04$ for AUMic (and $H / r \leq 0.02$ close to the position of maximum surface density). The $\beta$ Pictoris disc appears thicker with $H / r$ ratios as large as $\simeq 0.1$ (Golimowski et al. $2006)^{7}$. The modelling and inversion of scattered light brightness profiles of inclined, ring-shaped discs do not provide many more constraints. The HD 181327 rings for example, might have $H / r$ ratio as large as about 0.1 at the positions of maximum surface density, but the actual ratios could be two times smaller (Schneider et al. 2006).

There are thus large uncertainties, but it seems however that the 0.01 to 0.1 range is the most realistic one for $\langle i\rangle$ (and thus $\langle e\rangle$ if assuming equipartition). This $0.01-0.1$ range does also make sense when considering simple theoretical arguments regarding

\footnotetext{
6 The analytical derivation of Sect. 4 is invalid here, since it implicitly assumes that small high- $\beta$ grains dominate the total optical depth.

${ }^{7}$ However, these measurements include the so-called disc warp which, according to Golimowski et al. (2006), might be due to a blend of two separate, intrinsically thinner disc components inclined with respect to each other by a few degrees
} 
the sizes of the biggest objects within the disc. Consider indeed a belt of planetesimals sitting at $\sim 100$ AU from the central star. If the maximum size is limited to, say, $\sim 10 \mathrm{~km}$, then excitation by mutual viscous stirring leads to values of the order of their surface escape velocity $(\sim 10 \mathrm{~m} / \mathrm{s})$, and collisions among them generate particles with dispersion velocity that does not exceed the same velocity. The resulting $H / R \sim v_{\text {esc }} / v_{\text {kep }} \sim 0.003$ and is dynamically cold. On the other hand, if the maximum size is $\sim 500 \mathrm{~km}$, then $H / R \sim 0.15$ and the resulting debris disc is dynamically hot. This dynamically hot case probably makes more sense within the frame of the standard planet formation scenario, in which debris discs correspond to systems in which the bulk of planetesimal accretion process is already over and large planetary embryos are present (e.g. Kenyon \& Bromley 2005).

\subsection{Application to real systems}

As a consequence, we expect our nominal result, valid in the $\langle e\rangle \geq 0.01$ range, to correspond to the "natural" collisional evolution of most discs when left to themselves. This nominal model, with no sharp edge and luminosity falling as $r^{-3.5}$ compares well against the outer region $\mathrm{SB}$ profiles (beyond $\simeq 40 \mathrm{AU}$ and $\simeq 120$ AU respectively, see Table 1) for the two perhaps most emblematic debris discs: $\beta$ Pic and AU Mic. For these two systems, which are amongst the few ones for which (partial) disc thickness estimates are available, this result is in good agreement with the observed $\langle i\rangle$, which is for both discs in the $\geq 0.01$ range, and thus in the dynamically "hot" regime displayed in Fig. $1^{8}$. For AU Mic, this conclusion confirms the initial results obtained by Strubbe \& Chiang (2006) with a more simplified approach. For $\beta$ Pic, this result should be reinvestigated in more detail in a more complete study taking into account the numerous additional features observed in this system, but we believe our result to be robust regarding the global shape of this system's outer profile. Within the observational error bars, the nonimal result characteristics are also in relatively good agreement with outer disc profiles derived for several other debris discs, i.e. HD 53143 or HD 32297 (Table 1), especially when taking into account that strong anisotropic scattering could lead to somehow steeper profiles in $r^{-4}$ (Table 3).

For all these systems, no additional sculpting mechanism is needed in order to explain the main ring's edge and the profile beyond it. Of course, something had to sculpt the parent-body ring in the first place, but such a sculpting could have occurred in the past, when for instance gas was still present, and no longer be active today. Another alternative is that the birth-ring's outer edge corresponds to the natural radial limit at which large planetesimals can form in the protoplanetary nebulae.

There is however a group of systems (see Table 1) which have SB slopes in the -4.5 to -5 range (HD 139664, HD 107146, HD 181327), or even very sharp ring-like systems with brutal luminosity decreases in $r^{-6}$ or $r^{-7}$, represented by Fomalhaut and HR4796, which strongly depart from our nominal profile. Does this mean that these system's outer edges cannot be produced by "natural" internal collisional evolution and that "something else" is acting as a sculpting agent? The main question is here to see if these systems could fall into one of the categories giving alternative profiles presented in Sect. 3.2, especially the self-sustaining

\footnotetext{
${ }^{8}$ Our fit cannot give more quantitative information on the $\langle e\rangle$ and $\langle i\rangle$ dispersions, except that they are above the $\sim 0.01$ threshold value for the dynamically cold mode.
}

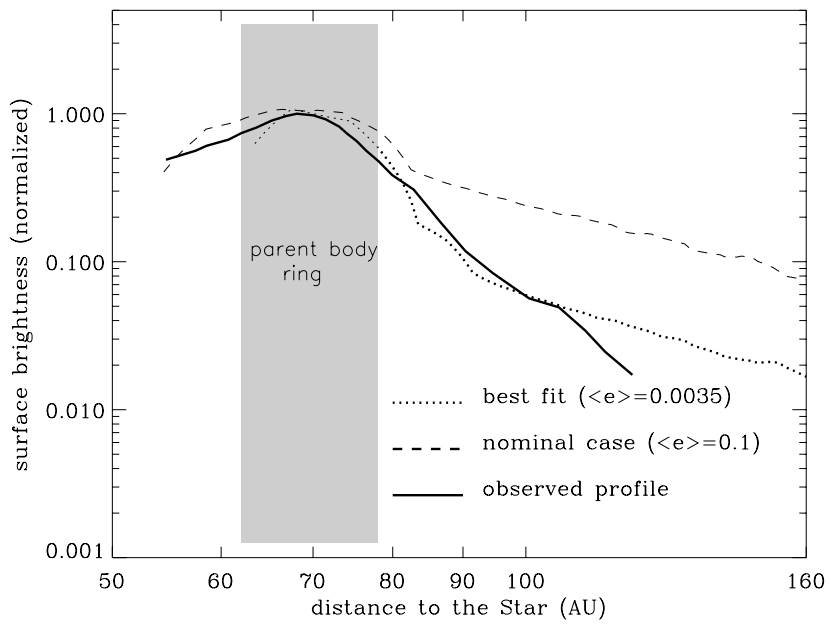

Fig. 12. The radial deprojected surface brightness profile for the HR4796 disc. Observed data are taken by Schneider et al. (1999) and are displayed in Fig. 6 of Wahhaj et al. (2005). Numerical profiles have been degraded to the resolution of the NICMOS/HST images, i.e., $0.12^{\prime \prime}=8 \mathrm{AU}$. The best numerical fit is obtained for $\Delta r_{\mathrm{BR}}=16 \mathrm{AU}$ and $\langle e\rangle=0.0035$.

dynamically cold case. As a representative example, we consider the specific case of HR4796 $\mathrm{A}^{9}$.

\subsection{Fitting a sharp edge ring: HR4796A}

As for most debris discs, there exists no fully reliable observational constraints on the vertical height and therefore dynamical excitation of the HR4796A disc. From model fitting of scattered light and IR images, Augereau et al. (1999) argue that the scale height at $70 \mathrm{AU}$ (the location of the main ring) has to be less than 7-8 AU, or $2\langle i\rangle \sim\langle e\rangle \leq 0.1$. However, from image fitting and theoretical constraints, Kenyon et al. (1999) find that the most likely value is $H \sim 0.5 \mathrm{AU}$, i.e. $2\langle i\rangle \sim\langle e\rangle \leq 0.007$. It is thus plausible that this system falls into the dynamically cold category.

We numerically explore a series of dynamically cold HR4796-like systems, fixing the ring's center at $70 \mathrm{AU}$ and taking the radial extent of the birth ring $\Delta r_{\mathrm{BR}}$ as well as $\langle e\rangle$ as free parameters. The observed profile comes from HST/NICMOS (Schneider et al. 1999) and is displayed in Fig. 6 of Wahhaj et al. (2005). Figure 12 shows the best fit obtained in our parameter exploration. It corresponds to a dynamically cold case with $\langle e\rangle=0.0035$ and a radial width of the birth ring $\Delta r_{\mathrm{BR}}=16 \mathrm{AU}$, which is close to the $\simeq 13-17$ AU derived from observations (Schneider et al. 1999; Schneider 2001) ${ }^{10}$. As a comparison, we also display a "nominal" profile obtained for $\langle e\rangle=0.1$. The latter is fully incompatible with the observed profile over the 80-120 AU domain by almost 2 mag in brightness. The best fit (dynamically cold case) compares well with the data in the 70-110 AU region and departs from the observed $S B(r)$ profile only between 110 and $120 \mathrm{AU}$, where the numerical profile becomes too flat. It is difficult to say how significant this discrepancy in these outermost $10 \mathrm{AU}$ is, as there are no error bars given

\footnotetext{
9 The imaging data on Fomalhaut do not cover regions beyond $20 \mathrm{AU}$ from the outer edge (Kalas et al. 2005), which is not enough to proceed to a reliable numerical fit.

${ }^{10}$ Note that since the disc's width is resolved in the near-IR (Schneider et al. 1999; Schneider 2001), it could in principle be taken as a fixed input in our model. However, we kept it as a free parameter to check the validity of our fit.
} 
in the Wahhaj et al. (2005) plot. It is possible that confusion from the sky background enters at these distances.

We must thus remain careful but it seems that there is at least a possibility for our dynamically cold model to provide an explanation for sharp outer edge discs like HR4796A. The question of how realistic this explanation can be is another issue. We have seen in Sect. 5.1.2 that the $\langle i\rangle<0.01$ condition, although probably not generic with respect to planet-formation scenarios, cannot, in most cases, be explicitly ruled out by observations.

There may however be a prediction made by the cold-disc scenario which could be observationally checked, i.e., the underabundance of grains in the $\mu \mathrm{m}$ to sub-millimetre range ${ }^{11}$.

For HR4796, for instance, Augereau et al. (1999) have performed detailed fits of the SED as well as of thermal and scattered light images. Their best fit implied that most of the geometrical cross section was contained in grains close to the minimum value for their size distribution, i.e. $s_{\min } \sim 10 \mu \mathrm{m}$. However, this fit was obtained assuming an imposed size distribution in $s^{-3.5}$, so that these results cannot be used to rule out the possibility of higher $s_{\min }$ values for alternate size distributions. Wahhaj et al. (2005) performed similar fits with partly more recent data and found that the effective size for grains within the ring is $\sim 50 \mu \mathrm{m}$. This would be in relatively good agreement with our dynamically cold case. However, this value was obtained assuming a single size for the dust population, an obvious limitation the authors are fully aware of as they acknowledge the need for "physically meaningful size distributions". It is a general characteristics of such global fits to have too many free parameters to constrain the dust composition as well as size and spatial distributions in an unambiguous nondegenerate way. They usually have to rely on some starting assumption regarding the size distribution. In this respect, it would be interesting to test these best fit models with alternate size distributions, as obtained from numerical collision-evolution studies, but this is an issue which exceeds by far the scope of the present work.

There exists however yet unpublished scattered light data (Schneider et al., http: //nicmosis . as . arizona . edu: 8000) indicating that the ring is uniformly red from $\mathrm{V}$ to $\mathrm{H}$. This should mean that the emission is dominated by grains in the sub- $\mu \mathrm{m}$ to $\mu \mathrm{m}$ range. Should this feature be confirmed, then the dynamically cold state could probably be ruled out. Note however that this puzzling feature would be very difficult to interpret for any dynamical model of the HR4796A ring. Indeed, it is not easy to explain how the luminosity, and thus the geometrical cross section could be dominated by unbound grains which should leave the ring on dynamical timescales ${ }^{12}$.

\section{Summary and conclusion}

We numerically investigate if the diverse outer surface brightness profiles observed in debris discs can be explained by the "natural" collisional evolution of belts made of solid particles ranging from planetesimal to micron-sized dust grains. We consider an initial ring of parent bodies with a razor-sharp outer edge and quantitatively examine to what extent the steady collisional production of small, radiation-pressure-affected grains modifies the initially perfect ring-like structure.

\footnotetext{
11 While this paper was being reviewed, Liseau et al. (2008) reported the detection of a dust ring around q Eri, which could possibly be very confined and depleted from $\leq 100 \mu \mathrm{m}$ grains.

12 This seems to be a recurrent issue which is not limited to HR4796A: vast populations of unbound particles could also dominate the scattered luminosity of HD 141569A (Augereau \& Papaloizou 2004), and might be present in the outer $\beta$ Pictoris disc (Augereau et al. 2001).
}

Concurring with the pioneering results of Strubbe \& Chiang (2006), our numerical explorations have shown that for most "reasonable" parameters (mass, ring width, dynamical excitation) of a collisionally evolving debris ring, the surface brightness outside the ring naturally tends towards a standard profile, with no sharp drop at the outer edge and a mid-plane surface brightness profile $\propto r^{-3.5}$. We confirm this result by simple but robust analytical considerations. This nominal result is in good agreement with the luminosity profiles observed in the outer regions of several debris discs, including $\beta$ Pictoris and AUMic.

Some observed systems however exhibit steeper radial luminosity profiles. Our numerical exploration shows that sharper outer edges and profiles steeper than that of the nominal case can only be obtained for two "extreme" cases:

1. For a system with very high dust content (typically $M_{\text {dust }} \geq$ $10 M_{\oplus}$ ), the radial optical depth is raised to near unity for small grains. Most of these high $\beta$ grains cannot travel out of the birth ring without suffering a collision. This allows the outer edge to sharpen. However, systems with such high radial optical depth are expected to wear down with time because of strong collisional erosion. Moreover, the existence of such very dusty systems is not backed by observations. This case does not appear as a realistic option.

2. A longer survival of the sharp outer edge is achieved for systems with normal dust content that are dynamically cold, with typically $\langle e\rangle=2\langle i\rangle \leq 0.01$. In this case, small grains are destroyed much more efficiently than they are created, leading to a depletion of this population. The system's optical depth and luminosity are then dominated by large grains which do not leave the main birth ring, leading to a sharp outer edge. Even if this case might not correspond to the most generic debris disc configuration, it cannot be ruled out by observations and is thus a possible explanation to some of the observed systems.

To numerically investigate the applicability of the dynamically cold case to real sharp-edge systems, we consider the specific case of HR4796A. We find a reasonably good fit of this system's outer region luminosity profile with a dynamical excitation $\langle e\rangle \sim 0.0035$. There is thus the possibility that such a sharp outer edge could be explained by the natural collisional evolution of a confined disc of large parent bodies.

Acknowledgements. P.T. wishes to thank Jean-Charles Augereau and Alexander Krivov for fruitful discussions.

\section{References}

Ardila, D. R., Golimowski, D. A., Krist, J. E., et al. 2004, ApJ, 617, L147

Artymowicz, P. 1997, Ann. Rev. Earth Planet. Sci., 25, 175

Augereau, J.-C., \& Beust, H. 2006, A\&A, 455, 987

Augereau, J. C., \& Papaloizou, J. C. B. 2004, A\&A, 414, 1153

Augereau, J. C., Lagrange, A. M., Mouillet, D., Papaloizou, J. C. B., \& Grorod, P. A. 1999 , A\&A, 348, 557

Augereau, J. C., Nelson, R. P., Lagrange, A. M., Papaloizou, J. C. B., \& Mouillet, D. 2001, A\&A, 370, 447

Backman, D. E., \& Paresce, F. 1993, Main-sequence stars with circumstellar solid material - The VEGA phenomenon, in Protostars and planets III (Tucson: Univ. of Arizona Press), 1253

Besla, G., \& Wu, Y. 2007, ApJ, 655, 528

Campo Bagatin, A., Cellino, A., Davis, D., Farinella, P., \& Paolicchi, P. 1994, Planet. Space Sci., 42, 1079

Dohnanyi, J. S. 1969, JGR, 74, 2531

Fitzgerald, M., Kalas, P., Duchêne, G., Pinte, C., \& Graham, J. 2007, ApJ, 670, 536

Freistetter, F., Krivov, A. V., \& Lohne, T. 2007, A\&A, 466, 389 
Golimowski, D. A., Ardila, D. R., Krist, J. E., et al. 2006, AJ, 131, 3109

Greaves, J. L. 2005, Science, 307, 68

Henyey, L. G., \& Greenstein, J. L. 1941, ApJ, 93, 70

Jayawardhana, R., Fisher, S., Hartmann, L., et al. 1998, ApJ, 503, 79

Jonkheid, B., Kamp, I., Augereau, J.-C., \& van Dishoeck, E. F. 2006, A\&A, 453, 163

Kalas, P. 2005, in Proceedings of the Miniworkshop on Nearby Resolved Debris Disks, ed. I. Kamp \& M. Meixner, 13

Kalas, P., Graham, J., \& Clampin, M. 2005, Nature, 435, 1067

Kalas, P., Graham, J., Clampin, M., \& Fitzgerald, M. 2006, ApJ, 637, 57

Kenyon, S. J., \& Bromley, B. 2005, AJ, 130, 269

Kenyon, S. J., Wood, K., Whitney, B. A., \& Wolff, M. J. 1999, ApJ, 524, 119

Klahr, H., \& Lin, D. 2005, ApJ, 632, 1113

Koerner, D. W., Ressler, M. E., Werner, M. W., \& Backman, D. E. 1998, ApJ, 503, L83

Krivov, A., Lohne, T., \& Sremcevic, M. 2006, A\&A, 455, 509

Lagrange, A.-M., Backman, D. E., \& Artymowicz, P. 2000, in Protostars and Planets IV, (Tucson: Univ. of Arizona Press), 639

Lecavelier des Etangs, A., Vidal-Madjar, A., \& Ferlet, R. 1996, A\&A, 307, 542

Liseau, C., Risacher, A., Brandeker, C., et al. 2008, A\&A, 480, L47
Lissauer, J., \& Stewart, G. 1993, in Protostars and Planets III (Tucson: Univ. of Arizona Press), 1061

Löhne, T., Krivov, A., \& Rodmann, J. 2008, ApJ, 673, 1123

Moro-Martin, A., \& Malhotra, R. 2005, ApJ, 633, 1150

Nakano, T. 1990, ApJ, 355, L43

Quillen, A. 2007a, MNRAS, 377, 1287

Quillen, A. 2007b, MNRAS, 380, 1642

Schneider, G. 2001, BAAS, 198, 83.01

Schneider, G., Smith, B. A., Becklin, E. E., et al. 1999, ApJ, 513, L127

Schneider, G., Silverstone, M. D., Hines, D. C., et al. 2006, ApJ, 650, 414

Smith, B., \& Terrile, R. 1984, Science, 226, 1421

Strubbe, L. E., \& Chiang, E. I. 2006, ApJ, 648, 652

Su, K. Y. L., Rieke, G. H., Misselt, K. A., et al. 2005, ApJ, 628, 487

Telesco, C. M., Fisher, R., Wyatt, M., et al. 2005, Nature, 433, 133

Thébault, P., \& Augereau, J. C. 2005, A\&A, 437, 141

Thébault, P., \& Augereau, J. C. 2007, A\&A, 472, 169

Thébault, P., Augereau, J.-C., \& Beust, H. 2003, A\&A, 408, 775

Wahhaj, Z., Koerner, D. W., Backman, D. E., et al. 2005, ApJ, 618, 385

Wyatt, M. C., Dermott, S. F., Telesco, C. M., et al. 1999, ApJ, 527, 918

Wyatt, M. C., Smith, R., Su, K. Y. L., et al. 2007, ApJ, 633, 365 